\title{
Vision-Based Parking Entry Control System
}

\author{
Shahrulnafiin Saharudin and Ahmad Ridhwan Wahap*
}

School of Electrical Engineering, Universiti Teknologi Malaysia, 81310 UTM Skudai, Johor, Malaysia.

"Corresponding author: aridhwanwahap@utm.my

\begin{abstract}
Vision-based control systems are very promising in the Intelligent Transportation System (ITS). This paper proposes a system that use the vision system to control vehicle entry at the bridge gate at a certain facility. The system detects the presence of the car and captures the front car image to proceed to plate recognition process. Vehicle plate region is extracted using the size filtering, image thresholding and object counting algorithms. Optical character recognition technique is used in the recognition module. The result from the recognition module is then compared to the record in the database for information like the vehicle owner name, type of car, etc. The overall system is implemented and simulated in LabVIEW and the performance of recognition is tested on the real image. The system can successfully detect and recognize the plate number with minimum error.
\end{abstract}

Keywords: Automatic Number Plate Recognition; LabVIEW vision; vehicle plate recognition; Optical character recognition.

(C) 2018 Penerbit UTM Press. All rights reserved

\section{INTRODUCTION}

License plate recognition system is the technology that uses the optical character recognition on images to recognize vehicle registration number. There are many different names for car license plate recognition system available in the market such as License Plate Recognition (LPR), Car Plate Recognition (CPR), Automatic License Plate Recognition (ALPR) and others. Although their names are different, their main objective is to detect the car license plate and recognize the numbers and the characters on the license plate. These license plate recognition systems can be applied to various places to provide extra security system and safety. The license plate recognition system has been introduced decades ago and keep evolving year by year. This system has been developed using various techniques and approaches to achieve the better performance in recognizing the license plate number. To successfully recognize the Malaysian license plate system, the use of proper approach need to be considered. Some researchers have proposed a localization method to detect the Malaysian license plate [1].

The ALPR system consists of three modules, the first module is the detection module to detect the region of the interest (ROI) from the input image, the second module is the extraction module to extract the plate number location from the ROI specified earlier, and the last module is the recognition module to recognize the character that has been segmented. The rest of the paper is organized as follows: section 2 methodology and devices used in the project. The result and discussion are analyzed in section 3 , followed by the conclusion in section 4 .

\section{PROJECT METHODOLOGY}

\subsection{General Flow Diagram}

The overall system flow is illustrated as below in Figure 1, starting with the input front car image to the system, then the images will go through the LP ROI detection, extraction and recognition module sequentially. The output from recognition module then will be compared to the local database to verify whether the car owner is a registered owner or not.

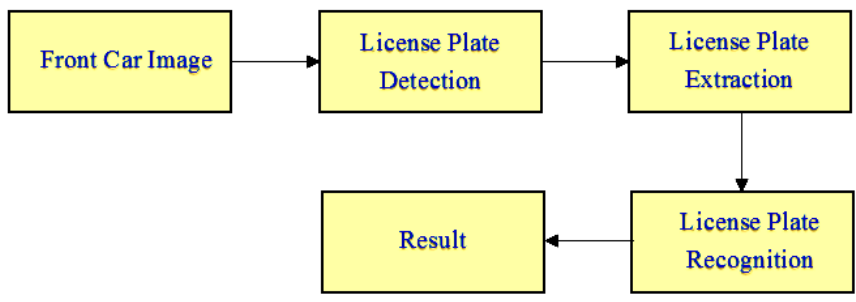

Figure 1. The overall system design

\subsection{Hardware Description}

There is no hardware works in this project because the project fully focused on the algorithm and software development only. Smartphone - iPhone camera is used to take the real picture of the front car on the bridge-gate entrance.

\subsection{Software}

\subsubsection{Operating System (Windows)}

There is no hardware works in this project because the project fully focused on the algorithm and software development only. Smartphone - iPhone camera is used to take the real picture of the front car on the bridge-gate entrance.

\subsubsection{Programming Software}

The programming software used for this project is LabVIEW software. LabVIEW is the graphical programming software that allows for control, data acquisition, and pre/post-processing of the acquired data [2]. LabVIEW software helps make the complexity of low-level programming and embedded hardware 
configuration invisible to the user, so the domain expert can focus on the design (algorithms, mathematical models, signal processing routines, and so on) instead of low-level software and hardware issues that are not the key subject matter of the research work.

NI Vision Assistant module of LabVIEW that provided step by step instruction for vision application. The algorithm is developed by using the module build-in feature.

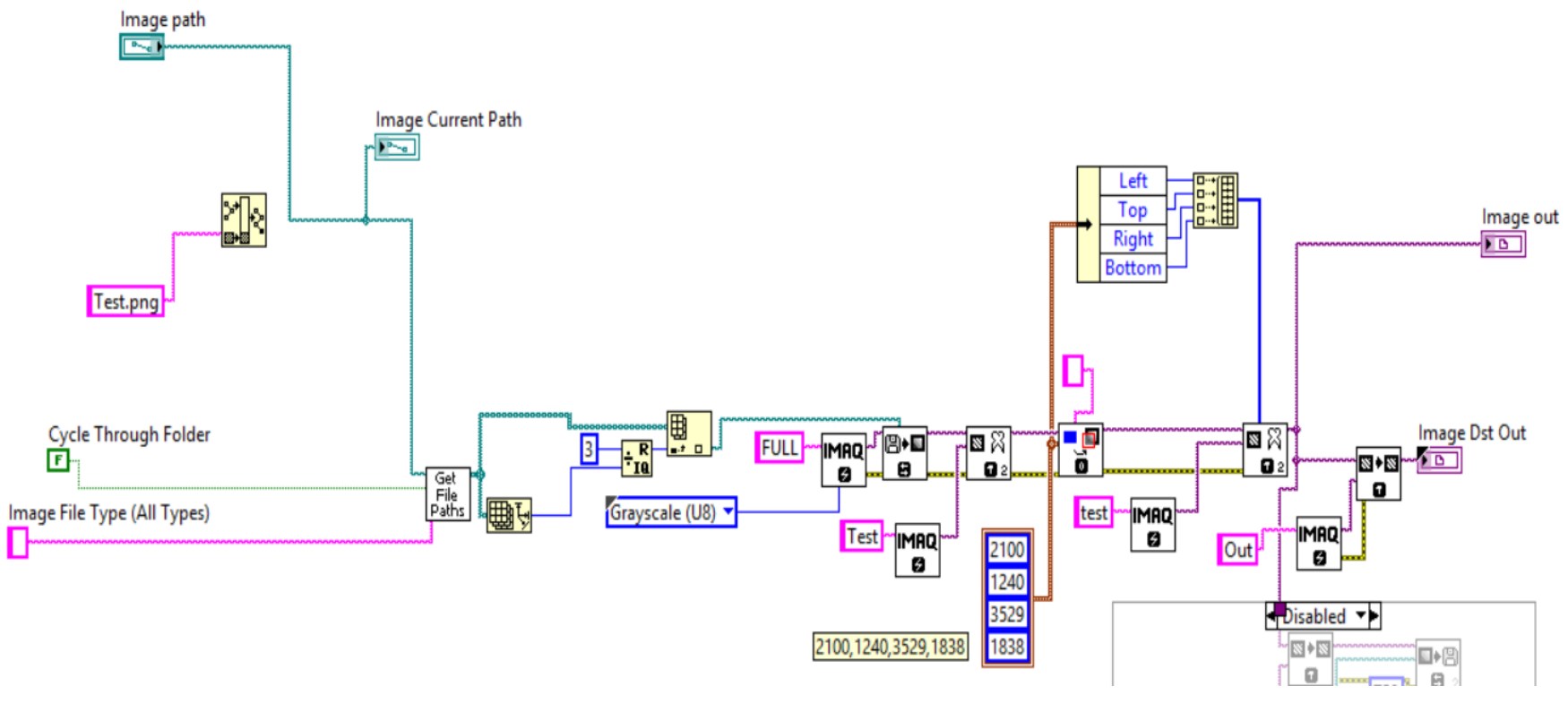

Figure 2. Block Diagram for detection module

\section{RESULTS AND DISCUSSION}

The car image is taken by using the high-resolution iPhone camera and in JPG image format. The resolution of the picture is $4032 \times 3024$ pixels.

\subsection{Detection Module}

The input car image goes through the detection module Vi that has been develop as shown in Figure 2 (due to space restriction, the image is located on the last section of the paper). This $\mathrm{Vi}$ extracts the image based on ROI specified in the algorithm from the original image to be used for extraction and recognition.

Output image from the detection module is shown in Figure 3. This image will be saved later on the local storage by naming it as "plate number".PNG. The picture has been set to grayscale image to make sure it is ready for the next module.

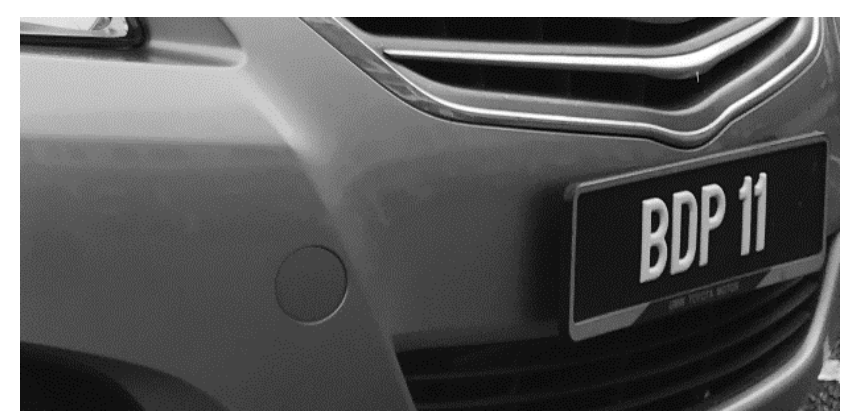

Figure 3. Output from Detection Module

\subsection{Extraction and Segmentation Module}

Step -I: Threshold-Select the threshold range of 122 to the dark object on the image. The result is stored in the image buffer to be used in the last process. The output should be as shown in Figure 4.

Step -II: Threshold -Select the threshold range of 152 looking for the bright image on the picture. The output image from this function is a binary image as shown in Figure 5.

Step -III: Advance Morphology -The image from the previous step need to be smoothed out to reduce the detection error. The operation can be done by selecting advance morphology function and choose remove border object to remove the border on the binary image. The output result is shown in Figure 6.

Step -IV: Advance Morphology -Output image need to go through the advance morphology one more time to remove the small particle on the image. This step is to ensure that we can ignore the small particles that should not include in the recognition process. To perform this operation, select the advance morphology function and select remove small particle option. Figure 7 shows the screen after applying the filter.

Step -V: Operator - The binary image output from the advance morphology need to be changed into binary image for recognition module. To perform this operation, simply select the operator function and divide with the output image from step (i). The clear black and white image will be the final output shown in Figure 8.

Step -VI: Object Count -To segment each character on Fig. 8 image, develop the block diagram to detect each object on the 
image. Then, we set the setting according to the suitable parameter to meet the required outcome. By using our defined setting such as pixel size filtering, set the threshold value to 128 , and reject border, plate numbers successfully be segmented by each character as shown in Figure 9 below. The complete module is tested using another image to verify the accuracy and performance.

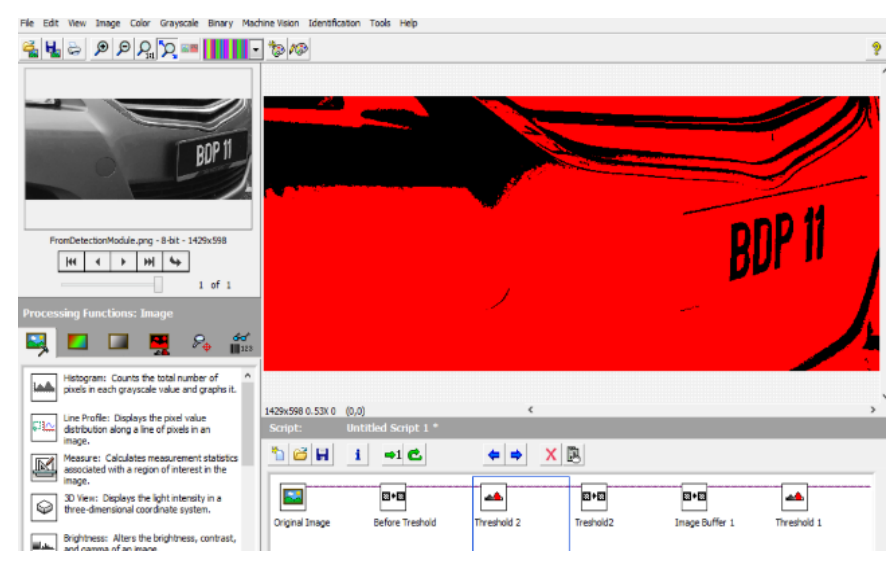

Figure 1. Screen after applying threshold value

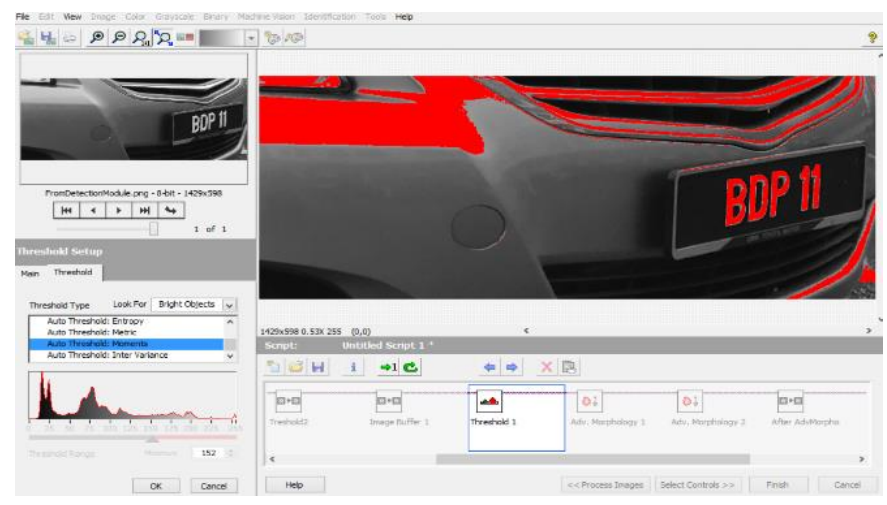

Figure 2. Screen after applying new threshold value

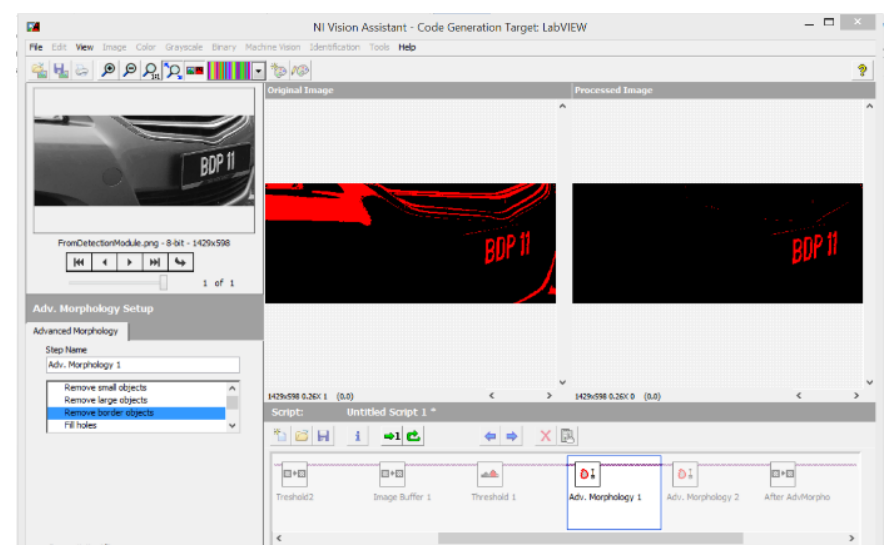

Figure 3. Screen after applying remove border

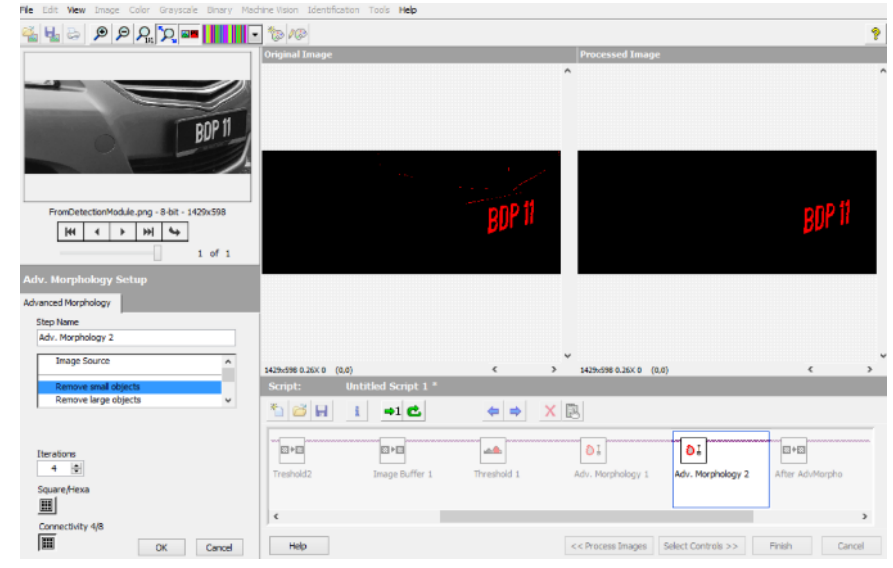

Figure 7. Screen after remove small object

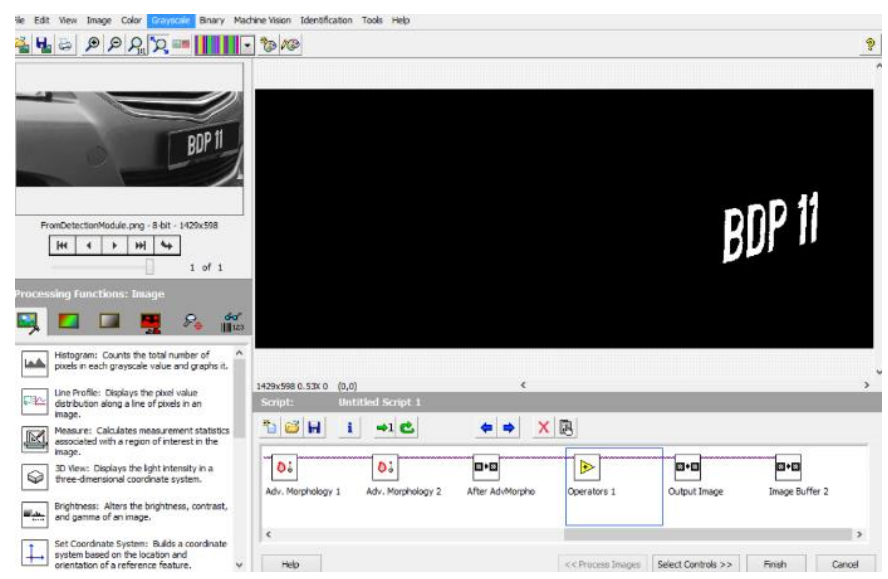

Figure 4. Screen after apply operator

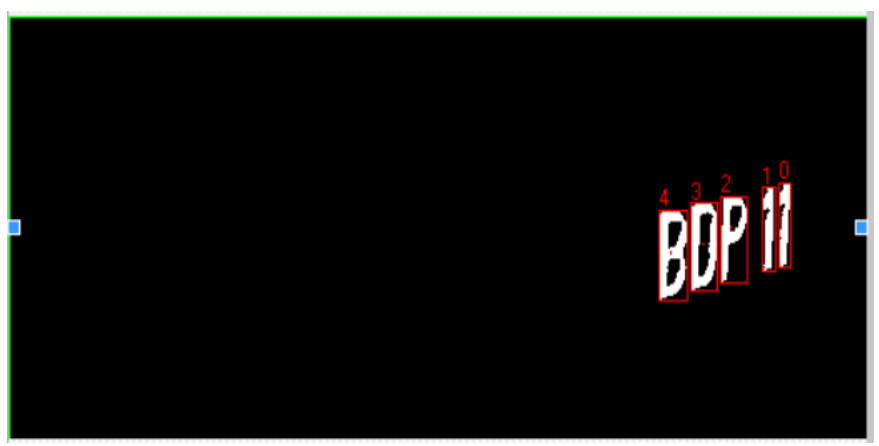

Figure 5. Output from extraction module

\subsection{Recognition Module}

For the last part of the module, the output from the extraction module need to be pre-processed before recognizing the segmented character on it. The pre-processing will remove all the unwanted object in the image. The character is then recognized by using Optical Character Recognition (OCR). OCR is the exact mechanisms that allow humans to recognize objects are yet to be understood, but the three basic principles are already well known by scientists - integrity, purposefulness, 
and adaptability [3]. The OCR need to be trained first before it can be used in the recognition system. The OCR is used to compare each individual character against the complete alphanumeric database. The OCR actually uses correlation method to match the individual character and finally is identified and stored format in a variable [4].

The output of this module is a string format of license plate number. The license plate string can be used to verify the car status with the database. The block diagram of the recognition module as shown in Figure 10.

\subsection{Database System}

The database system is something that is very important for an automation project like this. For this project, the local database system is used to store the car plate image and the detail of the owner. The detail of the owner is encrypted to prevent the information leaked to unauthorized personnel.

\subsection{Experimental Result}

Each module is combined to create one fully complete system that connected with the database system. The complete system represent as one simple user interface that can easy to understand by the user. The system then is tested using the front car image and Figure 11, Figure 12 and Figure 13 are the sample result from the system using three different front car image.

\section{CONCLUSION}

The vision-based parking entry system is used to automate the system at the parking entrance at the private facility by recognize the license plate number and verify with the database system for accessibility. The overall process really depends on the quality of the input image such as the lighting, contrast, brightness, resolution, and the camera position to capture the front car image. For this project, all image has been captured by using the same camera position and angle to make sure the accuracy of the recognition result. The software algorithm also maybe cannot process the image that captured during the rainy day. The accuracy and performance of this system are excellent because it gets about $90 \%$ correct license plate number recognition.

\section{REFERENCES}

[1] Tan, J. L., \& Abu-Bakar, S. R. (2012, November). A design method for Malaysian car plates character segmentation based on skew correction. In Control System, Computing and Engineering (ICCSCE), 2012 IEEE International Conference on (pp. 188-193). IEEE.

[2] Changalasetty, S. B., Badawy, A. S., Ghribi, W., \& Thota, L. S. (2014, April). Identification and feature extraction of moving vehicles in LabVIEW. In Communications and Signal Processing (ICCSP), 2014 International Conference on (pp. 1885-1889). IEEE.I.S. Jacobs and C.P. Bean, "Fine particles, thin films and exchange anisotropy," in Magnetism, vol. III, G.T. Rado and H. Suhl, Eds. New York: Academic, 1963, pp. 271-350.

[3] Kuldeepak, M. K., \& Vashishath, M. (2012). License Plate Recognition System based on Image Processing Using LabVIEW. International Journal of Electronics Communication and Computer Technology (IJECCT), 2(4).

[4] Qadri, M. T., \& Asif, M. (2009, April). Automatic number plate recognition system for vehicle identification using optical character recognition. In Education Technology and Computer, 2009. ICETC'09. International Conference on (pp. 335-338). IEEE.

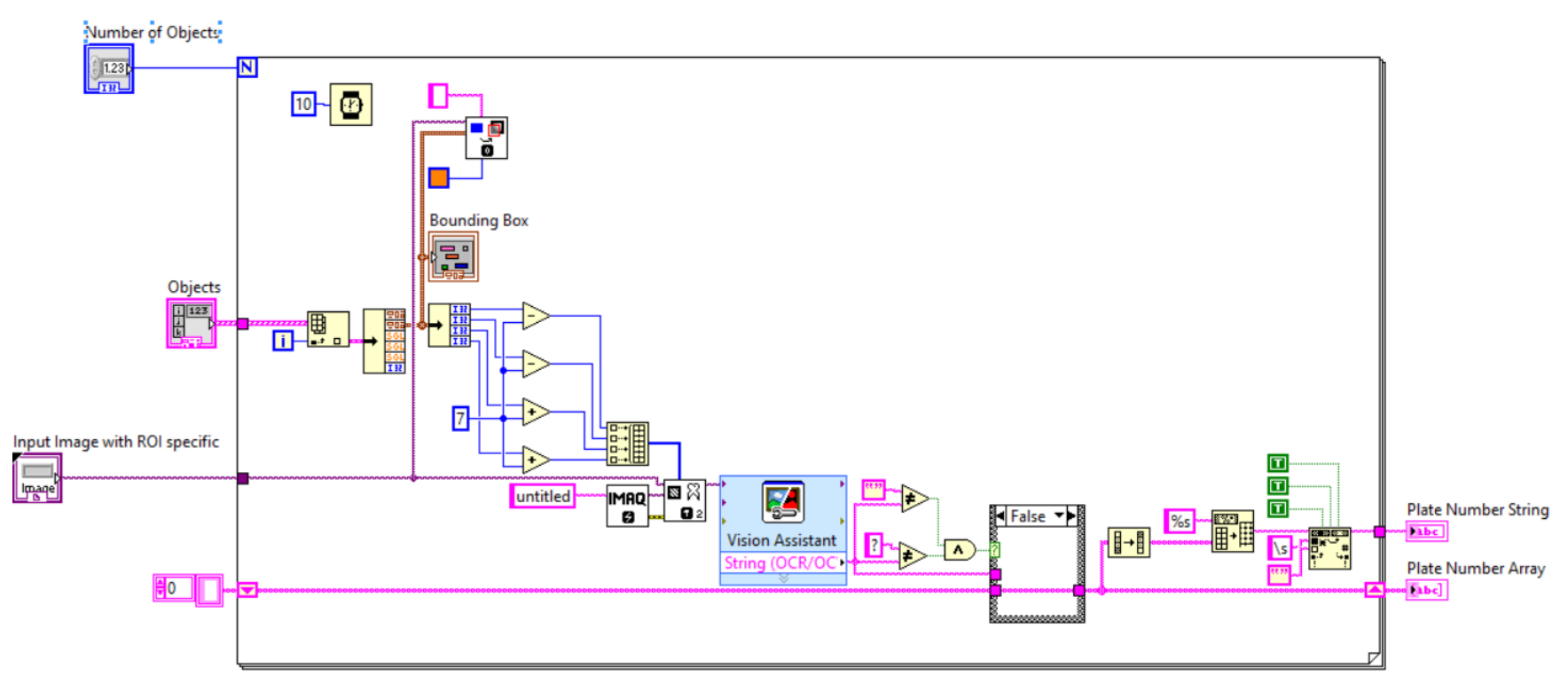

Figure 10. Block Diagram for recognition module 


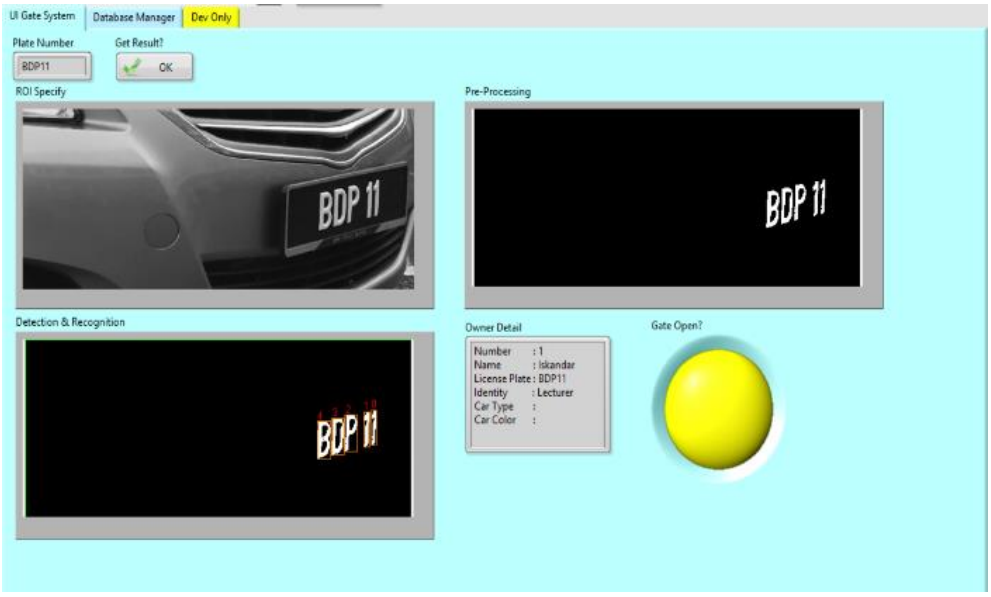

Figure 11. Result (1) from the complete system

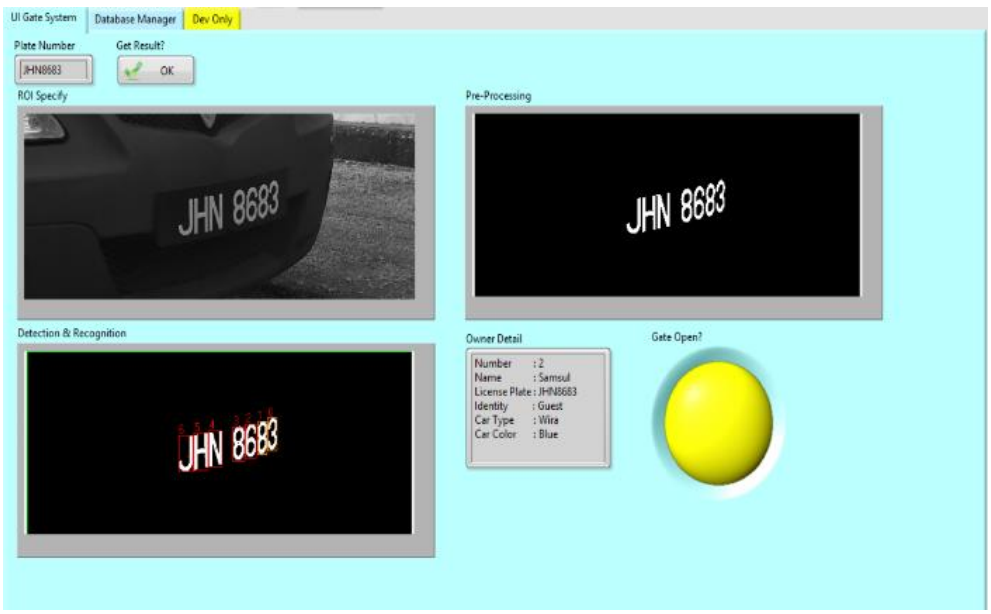

Figure 12. Result (2) from the complete system

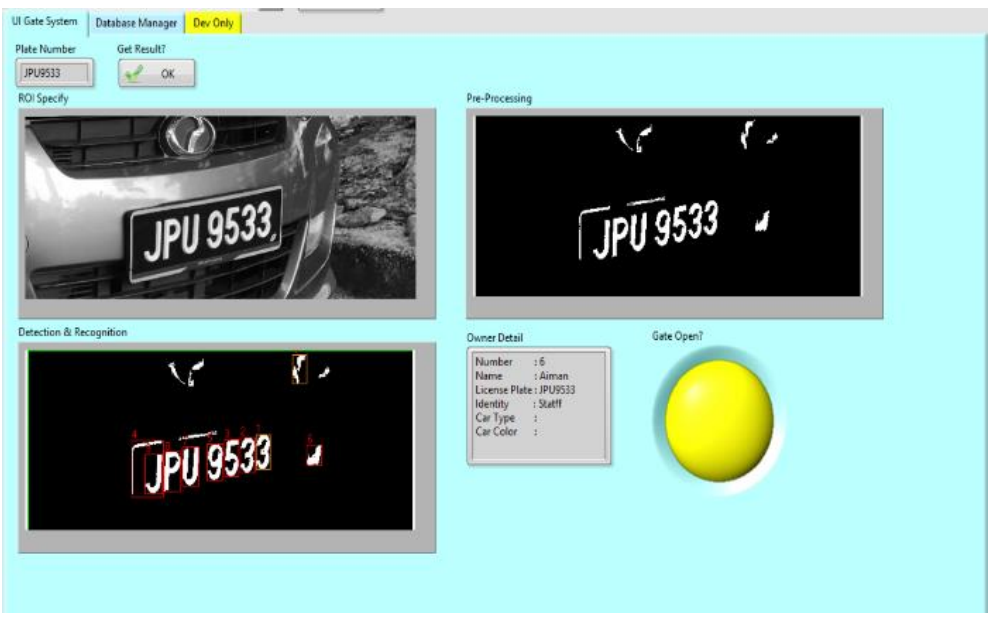

Figure 13. Result (3) from the complete system 